\title{
Correlação entre obesidade, adipocinas e sistema imunológico
}

Correlation between obesity, adipokines and the immune system

Resumo - A obesidade é considerada um problema de saúde mundial e o aumento de sua incidência, riscos e consequências são cada vez mais preocupantes. Essa enfermidade é caracterizada pelo acúmulo de tecido adiposo no organismo. Atualmente, muitos estudos estão direcionados à obesidade e as comorbidades associadas a esta patologia, visando esclarecer os mecanismos envolvidos. O tecido adiposo é um órgão dinâmico que secreta vários fatores, dentre eles as adipocinas. As adipocinas são peptídeos bioativos secretados pelos adipócitos e são importantes na regulação energética, resposta inflamatória e imunológica. Entre as adipocinas mais estudadas estão a leptina, adiponectina e resitina. O objetivo desta revisão foi reunir informações sobre as adipocinas estudadas (leptina, adiponectina e resistina) e suas relações com a resposta imunológica em indivíduos obesos, e a susceptibilidade desses pacientes à infecções. A partir dos dados encontrados na literatura algumas constatações podem ser realizadas. Os níveis circulantes dessas adipocinas estão diretamente associados ao grau de obesidade apresentado pelo indivíduo. Elevadas ou baixas concentrações circulantes das adipocinas estudadas podem acarretar benefícios ou prejuízos à competência imunológica, fazendo com que pessoas obesas sejam mais susceptíveis a infecções e inflamações que indivíduos eutróficos.

Palavras-chave: Obesidade; Adipocinas; Leptina; Adiponectina; Resistina; Sistema imunológico.

Abstract - Obesity is a worldwide health problem and the increase in its incidence, risks and consequences are a matter of growing concern. Obesity is characterized by the accumulation of fat in the body. Many studies are currently investigating obesity and associated comorbidities in an attempt to clarify the mechanisms involved. Fat tissue is a dynamic organ that secretes several factors, including adipokines. Adipokines are bioactive peptides secreted by fat cells, which are important for energy regulation and inflammatory and immune responses. Leptin, adiponectin and resistin are the most studied adipokines. The aim of this review was to gather information

1 Universidade Metodista de Piracicaba.

Piracicaba, São Paulo, Brasil.

Recebido em 01/09/08 Revisado em 10/12/08 Aprovado em 25/02/09 about these adipokines (leptin, adiponectin and resistin) and their relationship with the immune response in obese individuals, as well as the susceptibility of these patients to infections. The results of the literature review permit some observations. The circulating levels of these adipokines are directly involved in the degree of obesity of the patient. High or low circulating concentrations of these adipokines may have beneficial or negative effects on immune competence, with obese patients being more susceptible to infection and inflammation than eutrophic individuals.

Key words: Obesity; Adipokines; Leptin; Adiponectin; Resistin; Immune system. 


\section{INTRODUÇÃO}

Atualmente, a obesidade é um dos maiores problemas de saúde pública em muitos países, tanto pelo seu impacto na expectativa média de vida como pela piora na sua qualidade, sendo considerada uma epidemia global ${ }^{1}$.

Dados científicos comprovam a associação da obesidade a outras doenças como, por exemplo, diabetes, hipertensão, doenças cardiovasculares, dislipidemias, aterosclerose e algumas outras formas de câncer, representando, portanto, um grande problema para a saúde ${ }^{2}$. A obesidade se caracteriza pelo acúmulo de tecido adiposo localizado em todo o corpo, e se define como uma doença de prevalência crescente, sendo determinada pela associação de vários fatores: orgânicos, genéticos, ambientais, cultuais, alimentares e emocionais ${ }^{3}$.

O tecido adiposo é conhecido por possuir várias funções, entre elas: armazenamento de energia, regulação hormonal dos sistemas homeostáticos, termogênese e proteção de impacto contra as vísceras. Além disso, tem uma importante função endócrina, pois secreta uma variedade de proteínas sintetizadas e liberadas pelos adipócitos, denominadas adipocinas. Estas adipocinas têm diferentes funções, como: regulação de apetite e balanço energético, imunidade, sensibilidade à insulina, angiogênese, inflamação e resposta de fase aguda, pressão sanguínea e metabolismo de lipídeos ${ }^{4}$. Dentre as adipocinas mais conhecidas e estudadas no ambiente científico estão: leptina, adiponectina e resistina.

Não é fácil descrever a unidade funcional dessas adipocinas, porém, pode-se afirmar que muitas dessas adipocinas são ligadas à imunidade e inflamação, uma vez que foram estabelecidos paralelos (relações) entre os adipócitos e as células imunológicas ${ }^{5}$.

Dessa forma, o objetivo da presente revisão foi esclarecer associações da leptina, adiponectina e resistina com a obesidade (tecido adiposo) e suas relações com a resposta imunológica nesses indivíduos. Para tanto, realizou-se uma revisão literária de artigos nacionais e internacionais relacionados ao tema, nos portais científicos da Capes: Pubmed, Scielo e Highwire.

\section{LEPTINA}

Conhecida atualmente como "hormônio da saciedade", sendo o nome leptina derivado do grego leptos, que significa magro ${ }^{6}$. É um hormônio peptídico formado por 167 aminoácidos e peso molecular de $16 \mathrm{kDa}$, transcrito a partir do gene ob em camun- dongos. A mutação desse gene, ou sua deficiência, acarreta obesidade severa e diabetes tipo II nesses animais ${ }^{7,8,9}$.

A leptina é produzida pelo tecido adiposo e também por outros tecidos como placenta, medula óssea, estômago, músculo e talvez no cérebro. Além disso, é interessante observar que algumas citocinas como TNF- $\alpha$, IL-1 e IL-6 aumentam a expressão de RNAm para síntese de leptina ${ }^{7,9}$.

A leptina age através da ativação de receptores específicos classificados em seis isoformas (OBRa, OBRb, OBRc, OBRd, OBRe e OBRf), que são divididos em longos, curtos e solúveis ${ }^{6}$.

Uma das funções mais claras da leptina é ser um sinal aferente para o sistema nervoso central (SNC) através de uma retroalimentação negativa que inibe a expressão do gene da leptina, e posteriormente, controla a ingestão alimentar, regula o tecido adiposo, peso corporal e apetite ${ }^{6,8}$.

No hipotálamo, a leptina desenvolve um importante papel na regulação do balanço energético, atuando de duas maneiras. A primeira, estimulando a expressão de neuropeptídeos (anorexígenos) ligados aos mecanismos de inibição da ingestão alimentar (pro-ópio-melanocortina.- POMC e transcrito relacionado à cocaína e anfetamina - CART) e aumento do gasto energético total, através de inervação simpática ${ }^{6,8,9}$. E a segunda, inibindo a expressão do neuropeptídeo Y (NPY) e peptídeo agouti (AgRP), envolvidos nos mecanismos de aumento da ingestão alimentar e na redução do gasto energético, ${ }^{9,10}$.

A mediação da leptina na energia homeostática, ou seja, a liberação e captação energética são controladas pelas vias hipotalâmicas, enquanto outros efeitos são mediados por tecidos periféricos, incluindo músculo e células $\beta$ pancreáticas ${ }^{11}$. Quando a concentração de leptina diminui através do jejum ocorre a supressão do eixo- hipotalâmicopituitário-gonadal sinalizando a fome ${ }^{12}$. Uma exposição prolongada ao jejum diminui os níveis plasmáticos de leptina, ao passo que alimentação excessiva aumenta sua concentração. A leptina fica aumentada também por TNF- $\alpha$, estrogênio e diminuída com ácidos graxos livres e Hormônio do Crescimento $(\mathrm{GH})^{13}$.

Sugere-se que a leptina possui um papel modulador da resposta imune, atuando em processos inflamatórios e patologias imuno-mediadas. Em outros tecidos, a leptina possui funções metabólicas importantes como: secreção de insulina pelo pâncreas, produção de glicose hepática no fígado e captação de glicose pelo músculo ${ }^{14}$. 
Nos humanos, a expressão do gene ob está relacionada com o tamanho da massa de gordura corporal e é $75 \%$ mais alta em mulheres obesas que em homens obesos, o que sugere a influência de hormônios sexuais na sua regulação ${ }^{15}$. Assim, quanto maior a quantidade de tecido adiposo, maior a concentração de leptina produzida e liberada na circulação, uma vez que o percentual de gordura influencia nessa liberação?.

Existem vários mecanismos relacionados com a liberação de leptina tais como jejum, glicocorticóides, atividade simpática, insulina, exercício físico e alterações do balanço energético, que podem alterar as concentrações desse hormônio intrinsecamente associadas com a massa gorda ${ }^{8}$. Por outro lado, outros hormônios como os estrógenos, estimuladores, catecolaminas e andrógenos (testosterona) podem atuar inibindo a secreção da leptina ${ }^{16}$.

A leptina possui outros papéis importantes no organismo como: envolvimento na função reprodutiva, hematopoiese, angiogênese, resposta imune e formação óssea9. Algumas evidências mostram que a presença circulante elevada de leptina pode estar relacionada com a resistência dessa proteína na obesidade $^{8}$. Essa resistência pode estar ligada a um defeito no transporte de leptina ao sistema nervoso central. A resistência ocorre por dois motivos: o transporte saturado de leptina através da barreira hemato-encefálica e anormalidade na ativação do receptor de leptina e transdução de sinal.

A descoberta da leptina como um hormônio regulador do peso corporal possibilita encontrar soluções e favorecer as pessoas que têm dificuldade na perda de peso, já que ela leva informações sobre a quantidade de energia armazenada em forma de gordura para o cérebro (sinalizando a saciedade) e também determina mudanças no comportamento alimentar e gasto energético.

\section{LEPTINA E SISTEMA IMUNOLÓGICO}

Desde os últimos dez anos, estudos relacionam a leptina com o sistema imune, analisando seus efeitos imunomoduladores ${ }^{17}$.

A leptina possui efeitos pró-inflamatórios que previnem algumas doenças infecciosas ${ }^{5,18}$. No sistema imune, a leptina aumenta a produção de citocinas, a adesão e a fagocitose em macrófagos, além de estimular a proliferação das células $T$, levando ao aumento da competência imunológica ${ }^{9,17}$.

Em monócitos e macrófagos, a leptina aumenta a produção de citocinas pró-inflamatórias como TNF- $\alpha$, IL-6 e IL-12, e estimula a ativação de neutrófilos e a proliferação de monócitos in vitro circulantes ${ }^{5}$. O óxido nítrico sintetase (NOS) e espécies reativas de oxigênio (ROS), produzidos pela leptina, também induzem à ativação, proliferação e migração de monócitos circulantes 5 .

Essas evidências seriam sempre as mesmas, se os modelos de células utilizadas fossem de indivíduos obesos com deficiência genética a leptina, uma vez que por não existir defeitos nos receptores dessa adipocina, as células responderiam da mesma forma ao estímulo externo da leptina ${ }^{17}$. Porém, na forma mais comum da obesidade, em que ocorre hiperleptinemia, causada por deficiência genética associada a uma resistência à leptina, observa-se prejuízos variáveis na resposta imune, dos quais o mais preocupante se refere ao aumento no número de infecções ${ }^{17}$.

Nesse contexto, de acordo com Juge-Aubry et al. ${ }^{19}$ a insensibilidade do receptor de leptina é identificado pelas células $T$ como um estado de deficiência de leptina, o que induz a uma disfunção do sistema imunológico similar à produzida pela desnutrição ${ }^{19}$.

Shamsuzzaman et $\mathrm{al}^{20}$ investigaram 100 voluntários, homens e mulheres, e verificaram forte relação positiva entre leptina e Proteína-C-Reativa (PCR), concluindo que leptina e PCR desempenham papel de ligação entre mecanismos metabólicos, inflamatórios e doenças cardiovasculares ${ }^{20}$. Bulló et al. ${ }^{21}$ realizaram estudo com 100 sujeitos de ambos os sexos, e a análise de seus resultados sugerem que leptina e TNF- $\alpha$, aumentados na obesidade, podem induzir à produção de IL-6, PCR e outros fatores inflamatórios de fase aguda. Segundo os autores, esse estado inflamatório, considerado de baixo grau, pode contribuir para o aumento do risco de diabetes e doenças cardiovasculares, e embora a magnitude dessa inflamação seja baixa, a cronicidade desse baixo grau pode ser decisivo para a progressão da obesidade e de suas comorbidades ${ }^{21}$. Em outra pesquisa, Reilly et al. ${ }^{22}$ encontraram associação entre níveis de leptina e calcificação arterial coronariana, sugerindo que a leptina pode fornecer uma maior percepção de risco a pró-aterosclerose associado com a adiposidade ${ }^{22}$.

Analisando as evidências científicas citadas acima, podemos sugerir, através de hipótese, que o aumento dessa adipocina encontrado em indivíduos obesos causaria um comprometimento na resposta imunológica, fazendo com que esses sejam mais suscetíveis a doenças infecciosas e inflamações do que indivíduos eutróficos. 


\section{ADIPONECTINA}

A adiponectina é uma proteína plasmática de aproximadamente $30 \mathrm{kDa}$, relativamente abundante, que é secretada especificamente pelo tecido adiposo, sendo sua expressão maior no tecido adiposo subcutâneo do que no visceral ${ }^{18,23}$. Segundo Fantuzzi et al. ${ }^{5}$, a adiponectina circula em altas concentrações no sangue humano (bem mais alta comparada com a leptina) e possui várias atividades biológicas 5 .

Seus efeitos biológicos dependem não somente dos seus níveis circulantes na corrente sanguínea, mas também da especificidade tecidual e de seus receptores, ADP-R1 e ADP-R2 24 .

A adiponectina aumenta a oxidação muscular dos ácidos graxos e reduz a concentração de glicose plasmática através do AMPK (adenina monofosfato quinase $)^{18}$.

Sua síntese e secreção são reguladas por diversos mecanismos. Ao contrário da maioria das proteínas secretadas pelo tecido adiposo, a expressão de adiponectina diminui à medida que o tecido adiposo aumenta ${ }^{9,10,18,25}$.

Adipócitos pequenos secretam hormônios insulina-sensível, adiponectina, leptina e outros peptídeos. Adipócitos grandes induzidos por uma dieta gordurosa causam diminuição da produção de hormônios insulina sensíveis, resultando em resistência à insulina. Concentrações séricas de adiponectina são menores em indivíduos obesos com estado de resistência à insulina, uma vez que se correlacionam negativamente com a porcentagem de gordura corpórea, tolerância oral de glicose, insulina plasmática em jejum, além de fatores de risco cardiovascular, como pressão arterial, colesterol total, LDL-colesterol, triglicérides e ácido úrico ${ }^{23}$. Por outro lado, foi encontrada correlação positiva com a utilização de glicose durante clamp euglicêmico e com níveis HDL-colesterol ${ }^{26}$.

Isso mostra que o declínio da adiponectina acompanhado pelas mudanças apontadas, pode estar relacionado ao IMC e massa gorda do indivíduo, ou seja, as concentrações desse hormônio diminuem com a obesidade crescente. Outros fatores característicos que influenciam na concentração sérica dessa adipocina são sexo, idade, e estilo de vida. Mulheres possuem nível de adiponectina circulante maior do que homens, o que reflete um efeito androgênico ${ }^{25}$.

Além disso, a adiponectina possui efeitos antiaterogênicos, ou seja, demonstra a capacidade de inibir a adesão de monócitos ao endotélio vascular, a expressão de moléculas de adesão e também a expressão de TNF- $\alpha^{27}$.

\section{ADIPONECTINA E SISTEMA IMUNOLÓGICO}

Ao contrário de outras adipocinas, a adiponectina possui funções imunológicas anti-inflamatórias, pois age como proteção para fatores cardiovasculares e aumenta a sensibilidade à insulina ${ }^{5}$.

Algumas citocinas, como IL-6 e TNF- $\alpha$ são poderosos inibidores da secreção e expressão de adiponectina ${ }^{17}$. A adiponectina, por sua vez, regula a expressão de várias citocinas pró e antiinflamatórias, produzindo a IL-10 e IL-1, além de suprimir a síntese de TNF- $\alpha^{28}$. Além disso, inibe também a ativação do fator $\mathrm{kB}$ (NF-kB) em células endoteliais e interfere na função de macrófagos ${ }^{29}$. Assim, pode-se destacar que os níveis de adiponectina são inversamente correlacionados com os níveis de alguns mediadores inflamatórios, como IL-6, TNF- $\alpha$ e proteína-C- reativa ${ }^{17}$.

Existe uma correlação negativa entre adiponectina e PCR em humanos com aterosclerose. Essa associação negativa fortalece a hipótese da adiponectina como hormônio antagonizador do desenvolvimento de aterosclerose e inflamação vascular $^{27}$. Esse processo acontece, pois a adiponectina inibe a adesão dos monócitos ao endotélio, reduz a diferenciação mielóide, a produção de citocinas pelos macrófagos e a fagocitose ${ }^{17,27}$. Inibe, também, a produção e ação de TNF- $\alpha$ e suprime a transformação dos macrófagos em células espumosas (foam cells), isto é, a ligação entre inflamação vascular e aterosclerose $\mathrm{e}^{10,24}$.

A adiponectina, através da redução dos níveis de triglicérides no músculo e fígado, reduz a resistência à insulina em ratos obesos. Esse efeito é consequência do aumento de moléculas envolvidas tanto na metabolização de ácidos graxos, quanto na dissipação de energia muscular ${ }^{24}$. É importante destacar que a resistência à insulina em ratos lipoatróficos foi totalmente revertida com a combinação de ingestão de doses de adiponectina e leptina, mas, utilizando essas doses separadamente, esse quadro foi apenas parcialmente revertido ${ }^{30}$.

Essas evidências indicam uma possível utilização terapêutica, utilizando a adiponectina em alguns casos de doenças crônicas, principalmente, na resistência à insulina e diabetes tipo 2, com o objetivo de prevenir doenças e/ou diminuir os riscos associados a elas, como aterosclerose, porém mais estudos precisam ser realizados para esclarecer todos os mecanismos envolvidos. 


\section{RESISTINA}

A resistina pertence a uma família de proteínas ricas em cisteína, encontradas em regiões de inflamação, e representa a mais nova das adipocinas, descoberta em 200123,30.

É secretada por monócitos e adipócitos, possuindo cerca de 108 aminoácidos e peso molecular de $12.5 \mathrm{kDa}^{10,31}$.

Essa adipocina é expressa, especificamente, no tecido adiposo branco e sua secreção está fortemente relacionada à resistência à insulina ${ }^{10,23}$. Sua expressão é 15 vezes maior no tecido adiposo visceral, em comparação com o subcutâneo abdominal e glúteo-femora ${ }^{16}$.

Segundo alguns autores, em humanos, a expressão de resistina nos adipócitos é reduzida, e elevada nos macrófagos e monócitos, o que sugere um importante papel inflamatório ${ }^{5,18}$.

Os níveis de resistina aumentam na obesidade genética ou induzida por dieta e, portanto, estão ligados à resistência insulínica associada à obesidade. Seus efeitos no metabolismo da glicose são antagônicos aos da insulina, pois promovem resistência a esse hormônio por meio de aumento da glicogênese hepática, tendo rápido efeito sobre este tecido ${ }^{31}$.

A resistina regula, ainda, a diferenciação do adipócito por meio de mecanismo de retroalimentação negativa, que limita a formação do tecido adiposo em resposta a aumento do consumo de energia ${ }^{18}$.

Estudos feitos com roedores obesos demonstraram que a administração de anticorpos antirresistina diminuem a glicemia e melhoram a sensibilidade à insulina. Por outro lado, a administração adicional dessa adipocina resultou em efeitos contrários, como resistência à insulina e diminuição do transporte de glicose induzido pela insulina $^{32}$. Ainda assim, alguns autores sugerem que esses mecanismos relacionados à resistina, precisam ser melhor esclarecidos ${ }^{33}$.

Para Guimarães et al..$^{10}$, existe uma expectativa de que a identificação do receptor dessa adipocina e das vias de sinalização resultantes da alta ou baixa expressão de resistina em ratos transgênicos, possibilitem melhor entendimento sobre as funções biológicas dessa adipocina.

É importante ressaltar que em estudos com humanos os resultados ainda não estão totalmente esclarecidos. Não foi identificada a expressão gênica da resistina em humanos magros, enquanto que, em alguns estudos com obesos, ela foi identificada, porém não foi verificada nenhuma correlação com a massa corporal, adiposidade e resistência à insulina ${ }^{32,34,35}$. Outros estudos, no entanto, demonstraram correlação positiva entre os níveis de resistina circulante e o IMC $\mathrm{IM}^{35,36}$.

\section{RESISTINA E SISTEMA IMUNOLÓGICO}

Em humanos, a principal fonte de resistina surpreendentemente não é o adipócito, mas os macrófagos e monócitos, o que sugere um importante papel inflamatório ${ }^{5}$. Apesar de expressa e secretada em indivíduos magros, seus níveis estão comumente mais elevados na obesidade ${ }^{24}$.

A resistina possui grande ação aterogênica pelo aumento da expressão de moléculas de adesão intercelular-1 e antivascular-1 em células endoteliais vasculares e também pelo aumento da atividade do fator NF-kB, sinalizador para indução de adesão destas moléculas ${ }^{31}$.

Embora a resistina tenha sido primeiramente postulada como contribuinte para a resistência à insulina, cada vez mais evidencias indicam, também, que essa adipocina está envolvida no processo inflamatório. Alguns agentes pró-inflamatórios como TNF- $\alpha$, IL-6 e lipopolissacarídeos podem regular a expressão do gene da resistina ${ }^{37}$.

Um estudo de Kunnari et al..$^{38}$ mostrou que fatores inflamatórios são mais importantes na determinação da concentração plasmática de resistina do que valores da glicose e insulina. A resistina plasmática foi correlacionada com a razão cintura/ quadril, leucócitos do sangue, leptina, colesterol e pressão arterial diastólica.

Outra evidência que liga a resistina à inflamação é que os níveis de resistina plasmáticos foram associados com vários marcadores inflamatórios em algumas condições patológicas ${ }^{36}$. Estudos mostram que as pessoas que apresentavam sinais severos de inflamação apresentaram concentrações significantemente mais altas de resistina que indivíduos sadios. Nas pessoas com sinais inflamatórios severos, foi encontrada uma correlação positiva entre a resistina e os marcadores inflamatórios. Reilly et al. $^{22}$ encontraram correlação entre os níveis de resistina e marcadores de inflamação, como TNF- $\alpha$, IL-6, lipoproteína associada a fosfolipase A2, investigando 879 indivíduos ${ }^{22}$. Nesse estudo, os pesquisadores também observaram relação positiva entre a concentração de resistina e o índice de calcificação de artéria coronariana, concluindo que a resistina pode representar um novo elo entre sinais metabólicos, inflamação e aterosclerose. Kaser et al ${ }^{39}$ demonstraram em sua pesquisa que a 
expressão de RNAm de resistina encontra-se fortemente aumentada da mesma maneira que fatores pró-inflamatórios como TNF- $\alpha$, IL-1 e IL-6, sugerindo que os níveis desse hormônio, aumentados em humanos, podem ser uma ligação entre o processo inflamatório e resistência à insulina ${ }^{39}$. Em pesquisa realizada por Ohmori et al. ${ }^{40}$ foi encontrada associação dos níveis séricos de resistina com a presença e severidade de doença arterial coronariana, e também com resistência à insulina, confirmando, também, a hipótese da resistina desempenhar um papel de ligação entre a resistência insulínica, inflamação e doença arterial coronariana.

O envolvimento da resistina no processo inflamatório crônico, associado à obesidade, constitui hipótese alternativa capaz de justificar a presença dessa proteína, integrante de uma família de proteínas encontradas em regiões inflamatórias, no tecido adiposo de indivíduos obesos ${ }^{10}$.

\section{CONSIDERAÇÕES FINAIS}

Existem várias evidências científicas que demonstram a relação das adipocinas (leptina, adiponectina e resistina) com a obesidade e seu envolvimento na resposta imunológica. Esta revisão constatou, na literatura científica, a influência da obesidade nas concentrações das adipocinas estudadas, e suas relações com o sistema imune. Muitas pesquisas demonstraram que o grau de obesidade influencia diretamente nos níveis circulantes desses hormônios, sendo que a maioria delas apresentou correlação positiva entre obesidade e leptina, correlação negativa entre obesidade e adiponectina, e resultados bastante controversos para correlação entre resistina e obesidade, necessitando, assim, de mais pesquisas nessa área.

Recentes estudos destacam o envolvimento dessas adipocinas com a resposta imunológica, sugerindo que o aumento dos níveis de leptina e resistina, em indivíduos obesos, acarreta prejuízos na resposta imunológica. Segundo as evidências estudadas, indivíduos obesos que apresentam maior concentração de leptina e resistina são mais suscetíveis à doenças infecciosas e inflamatórias que indivíduos normais. Por outro lado, a adiponectina possui efeitos anti-inflamatórios, mas por apresentar correlação negativa com o tecido adiposo, em indivíduos obesos, a sua baixa concentração pode não ser suficiente para gerar melhoras consideráveis na competência imunológica.

Novos estudos precisam ser realizados com a finalidade de esclarecer os mecanismos envolvidos entre obesidade, adipocinas e sistema imunológico, uma vez que esta patologia é multifatorial e suas relações extremamente complexas. $\mathrm{O}$ entendimento dessas relações será de grande importância para a pesquisa de novos tratamentos que buscam uma melhora da saúde e da qualidade de vida de indivíduos obesos.

\section{REFERÊNCIAS BIBLIOGRÁFICAS}

1. World Helth Organization. Diet, nutrition and prevention of chronic diseases. Geneva; 2003,160.

2. Racette SB, Deusinger SS, Deusinger RH. Obesity: overview of prevalence, etiology, and treatment. Phys Ther 2003;83(3):276-288.

3. Villela NB, Neto OB, Curvello KL, Paneili BE, Seal C, Santos D, et al. Quality of life of obese patients submit ted to bariatric surgery. Nutr Hosp 2004;19(6):367-371.

4. Mafra D, Farage NE. O papel do tecido adiposo na doença renal crônica. J Bras Nefrol 2006;28(2):108-113.

5. Fantuzzi G. Adipose tissue, adipokines and inflammation. J Allergy Clin Immunol 2005;115(5):911-919.

6. Benatti FB, Lancha JR, AH. Leptina e exercício físico aeróbio: implicações da adiposidade corporal e insulina. Rev Bras Med Esporte 2007;13(4):263-269.

7. Mota GR, Zanesco A. Leptina, ghrelina e exercício físico. Arq Bras Endocrinol Metab 2007;51(1):25-33.

8. Negrão AB, Licínio J. Leptina: diálogo entre adipócitos e neurônios. Arq Bras Endocrinol Metab 2000;44(3):205-214.

9. Fonseca-Alaniz mh, Takada j, alonso-Vale MIC, Lima FB. O tecido adiposo como centro regulador do metabolismo. Arq Bras Endocrinol Metab 2006;50(2):216-229.

10. Guimarães DED, Sardinha FLC, Mizurini DM, Tavares do Carmo MG. Adipocitocinas: uma nova visão do tecido adiposo. Rev Nutr 2007;20(5):549-559.

11. Bjorbaek C, Kahn BB. Leptin signaling in the central nervous system and the periphery. Recent Prog Horm Res 2004;59(1):305-331.

12. El-Haschimi K, Pierroz DD, Hileman SM, Bjorbaek C, Flier J. Two defects contribute to hypothalamic leptin resistance in mice with diet-induced obesity. J Clin Invest 2000;105(12):1827-1832.

13. Margetic S, Gazzola C, Pegg GG, Hill RA. Leptin: a review of its peripheral actions and interactions. Int J Obes Relat Metab Disord 2002;26(11):1407-1433.

14. La Cava A, Matarese G. The weight of leptin in immunity. Nat Rev Immunol 2004;4(5):371-379.

15. Kleinubing MC. Ações dos níveis plasmáticos de leptina e sua influência no controle do peso corporal. Revista Digital EF Deportes: Buenos Aires. 2003; Disponível em: $<$ http://www.efdeportes.com/efd64/leptina. htm> [2008 jun 04].

16. Heermsdorff HHM, Vieira MAQM, Monteiro JBR. Leptina e sua influência na patofisiologia de distúrbios alimentares. Rev Nutr 2006;19(3):369-379.

17. Alvez MNR. Os efeitos da obesidade na resposta imune. Rev Bras Nutr Clin 2006;21(4):316-319. 
18. Costa JV, Duarte JS. Tecido adiposo e adipocinas. Acta Med Port 2006;19(3):251-256.

19. Juge-Aubry CE, Meier CA. Immunomodulatory actions of leptin. Mol Cell Endocrinol 2002;194(1-2):1-7.

20. Shamsuzzaman ASM, Winnicki M, Wolk R, Svatikova A, Phillips BG, Davison DE, et al. Independent association between plasma leptin and c-reactive protein in healthy humans. Circulation 2004;109(18):2181-2185.

21. Bulló M, Lorda PG, Megias I, Salas-Salvado J. Systemic inflammation, adipose tissue tumor necrosis factor, and leptin expression. Obes Res 2003;11(4):525-531.

22. Reilly MP, Iqbal N, Schutta M, Wolfe ML, Scally M, Localio AR, et al. Plasma leptin levels are associated with coronary atherosclerosis in type 2 diabetes. J Clin Endocrinol Metab 2004;89(8):3872-3878.

23. Carvalho MH, Colaço AL, Fortes ZB. Citocinas, disfunção endotelial e resistência à insulina. Arq Bras Endocrinol Metab 2006;50(2):304-312.

24. Fonseca-Alaniz MH, Takada J, Alonso-Vale MIC, Lima FB. O tecido adiposo como órgão endócrino: da teoria à prática. J Pediatr 2007;83(5):192-203.

25. Greenberg AS, Obin M. Obesity and the role of adipose tissue in inflammation and metabolism. Am J Clin Nutr 2006;83:461S-465S.

26. Matsuzawa y, Funahashi T, Kihara S, Shimomura I. Adiponectin and metabolic syndrome. Arterioscler Thromb Vasc Biol 2004;24(1):29-33.

27. Goldstein BJ, Scalia R. Adiponectin: a novel adipokine linking adipocytes and vascular function. J Clin Endocrinol Metab 2004;89(6):2563-2568.

28. Tilg H, Moschen AR. Adipocytokines: mediators linking adipose tissue, inflammation and immunity. Nature 2006;6(10):772-783.

29. Ouchi N, Kihara S, Arita Y, Maeda K, Kuriyama H, Okamoto Y, et al. Novel modulator for endothelial adhesion molecules: adipocyte-derived plasma protein adiponectin. Circulation 1999;100(25):2473-2476.

30. Carvalheira JBC, Zecchin HG, Saad MJA. Bases moleculares e fisiológicas da resistência à insulina. Hipertensão 2002;5(1):33-38.

31. Hermsdorff HHM, Josefina Monteiro JBR. Gordura visceral, subcutânea ou intramuscular: onde está o problema? Arq Bras Endocrinol Metab 2004;48(6):803-811.

32. Janke J, Engeli S, Gorzelniak K, Luft CF, Sharma AM.
Resistin gene expression in human adipocytes is not related to insulin resistance. Obes Res 2002;10(1):1-5.

33. Yamauchi T, Kamon J, Minokoshi Y, Ito Y, Waki H, Uchida S, et al. Adiponectin stimulates glucose utilization and fatty-acid oxidation b activating AMP-activated protein kinase. Nat Med 2002;8(11):1288-1295.

34. Iqbal N, Seshadri P, Stern L, Loh J, Kundu S, Jafar T, et al. Serum resistin is not associated with obesity or insulin resistance in humans. Eur Rev Med Pharmacol Sci 2005;9(3):161-165.

35. Azuma K, Katshkawa F, Oguchi S, Mitsuru, M, Yamazaki H, Shimada A, et al. Correlation between serum resistin level and adiposity in obese individuals. Obes Res 2003;11(8):997-1001.

36. Degawa-Yamauchi M, Bovenkerk JE, Juliar BE, Watson W, Kerr K, Jones R, et al. Serum resistin (FIZZ3) protein is increased in obese humans, J Clin Endocrinol Metab 2003;88(11):5452-5455.

37. Pang S, Le Y. Role of resistin in inflammation and inflammation-related diseases. Cell Mol Immunol 2006;3(1):29-34.

38. Kunnari A, Ukkola O, Paivansalo M, Kesaniemi A. High plasma resistin level is associated with enhanced highly sensitive c-reactive protein and leukocytes. J Clin Endocrinol Metab 2006;91(7):2755-2760.

39. Kaser S, Kaser A, Sandhofer A, Ebenbichler CF, Tilg H, Patsch JR. Resistin messenger-RNA expression is increased by pro-inflammatory cytokines in vitro. Biochem Biophys Res Commun 2003;309(2):286-290.

40. Ohmori R, Momiyama Y, Kato R, Taniguchi H, Ogura $\mathrm{M}$, Ayaori M, et al. Associations between serum resistin levels and insulin resistance. J Am Coll Cardiol 2005;46(2):379-380.

\section{Endereço para correspondência}

Cláudia Regina Cavaglieri

Educação Física, Faculdade de Ciências da Saúde,

Campus Taquaral, UNIMEP.

Rodovia do Açúcar, Km 156.

13400-911 - Piracicaba, SP. Brasil

E-mail: ccavagli@unimep.br 